\title{
Weaning of extracorporeal membrane oxygenation using continuous hemodynamic transesophageal echocardiography
}

\author{
Nicholas C. Cavarocchi, MD, ${ }^{\mathrm{a}}$ Harrison T. Pitcher, MD, ${ }^{\mathrm{a}}$ Qiong Yang, MD, ${ }^{\mathrm{a}}$ Pawel Karbowski, MS, ${ }^{\mathrm{a}}$ \\ Joseph Miessau, MS, ${ }^{a}$ Harold M. Hastings, $\mathrm{PhD},{ }^{\mathrm{b}}$ and Hitoshi Hirose, $\mathrm{MD}^{\mathrm{a}}$
}

\begin{abstract}
Background: Venoarterial extracorporeal membrane oxygenation (VA ECMO) has been used for profound cardiogenic shock to bridge to decision, ventricular assist device(s) (VADs), or transplant. To assess ventricular function and volume status along with hemodynamics during ECMO weaning, we developed a standardized weaning protocol, guided by a miniaturized transesophageal echocardiography probe designed for continuous hemodynamic monitoring (hemodynamic transesophageal echocardiography [hTEE]). We reviewed our experience with this weaning protocol with hTEE guidance to assess if we could predict patient outcomes.
\end{abstract}

\begin{abstract}
Methods: During the academic year of 2011, hTEE-guided ECMO weaning was performed in 21 patients on VA ECMO. Left and right ventricular function and volume status were assessed by continuous hTEE, while attempting to wean ECMO after a standardized protocol. The clinical outcomes, management, and positive predictive value of the device were investigated and analyzed for this cohort of patients.
\end{abstract}

Results: Of the 21 patients, 6 (29\%) had left and right ventricular recovery and underwent optimal medical therapy or revascularization for underlying coronary artery disease; $7(33 \%)$ had nonrecoverable left and right ventricular function; and $8(38 \%)$ had right ventricular recovery without improvement of the left ventricular function. These 8 patients underwent left VAD placement; none subsequently developed profound right ventricular failure. The positive predictive value for ventricular recovery by hTEE was $100 \%$ using our standardized ECMO weaning protocol (95\% confidence interval, $73 \%-100 \%)$.

Conclusions: The hTEE-guided ECMO weaning protocol accurately predicted the ability to wean ECMO to decision. This protocol can be applied by cardiac intensivists as a part of standard bedside intensive care unit assessment. (J Thorac Cardiovasc Surg 2013;146:1474-9)

Patients presenting with acute cardiogenic shock may require urgent placement of venoarterial extracorporeal membrane oxygenation (VA ECMO), before adequate assessment of either left or right ventricular function. VA ECMO at full flow provides near-total biventricular support and decompression of the heart, while allowing time to recover other end-organ function and to plan further medical or surgical management. Surgical management may involve implantable ventricular assist device(s) (VADs) or heart transplantation.

Determination of the left ventricular (LV) or right ventricular (RV) function before ECMO weaning is essential to predict biventricular or univentricular recovery

From the Department of Surgery, ${ }^{\text {a }}$ Thomas Jefferson University, Philadelphia, Pa; and the Department of Physics, ${ }^{\text {b }}$ Hofstra University, Hempstead, NY, and ImaCor, Garden City, NY.

Disclosures: Harold Hastings, $\mathrm{PhD}$, is an employee and stockholder in ImaCor, Inc. The other authors have nothing to disclose with regard to commercial support.

Received for publication March 5, 2013; revisions received June 6, 2013; accepted for publication June 28, 2013; available ahead of print Aug 29, 2013.

Address for reprints: Hitoshi Hirose, MD, Department of Surgery, Thomas Jefferson University, 607 College Bldg, 1025 Walnut St, Philadelphia, PA 19107 (E-mail: Hitoshi.Hirose@jefferson.edu).

0022-5223/\$36.00

Copyright (c) 2013 by The American Association for Thoracic Surgery

http://dx.doi.org/10.1016/j.jtcvs.2013.06.055 and possible subsequent left VAD (LVAD) placement. Ideal candidates for LVAD placement would be those who have isolated LV failure with reasonably recovered RV function. Lack of recognition of significant coexisting RV dysfunction may significantly increase the postoperative morbidity and mortality in those patients transitioned to LVAD placement, requiring prolonged inotropic agents, biventricular device support, or extracorporeal support.

Because of the physiologic features of VA ECMO, the ECMO circuit creates negative pressure and drains venous blood from the right atrium, preventing determination of ventricular function from cardiac output or mixed venous oxygen analysis using a Swan-Ganz catheter (Edwards, Irvine, Calif). Therefore, intensivists and surgeons have been looking for alternative methods to assess left and right ventricular function to make clinical decisions regarding ECMO weaning and possible subsequent LVAD placement.

A relatively recent tool allowing the intensivist to directly assess both the contractility and filling status of both the RV and LV at the bedside in real-time is a miniaturized hemodynamic transesophageal echocardiography probe (hTEE; ImaCor, Garden City, NY). Unlike conventional transesophageal echocardiography (TEE) probes, which are 10 to $15 \mathrm{~mm}$ in diameter and require a TEE-certified cardiologist to introduce the probe to the patient, the 


\section{Abbreviations and Acronyms \\ $\mathrm{ECMO}=$ extracorporeal membrane oxygenation \\ hTEE = hemodynamic transesophageal echocardiography \\ ICU = intensive care unit \\ $\mathrm{LV}=$ left ventricular \\ LVAD $=$ left ventricular assist device \\ $\mathrm{RV} \quad=$ right ventricular \\ TTE $=$ transthoracic echocardiography \\ $\mathrm{VA}=$ venoarterial \\ $\mathrm{VAD}=$ ventricular assist device}

hTEE probe is $5.5 \mathrm{~mm}$ in diameter, is disposable, and can remain indwelling for up to 72 hours for cardiac monitoring. ${ }^{1}$ The probe can be placed by intensivists with hTEE training, providing clinically useful information..$^{2-4}$ The diagnostic yield of hTEE is comparable to that of conventional TEE regarding biventricular functions and volume status. $^{2,3,5,6}$ The safety of TEE was well established, with a reported incidence of major complications of $0.2 \%$ to $0.5 \%$ per insertion..$^{7-10}$ It is reasonable to expect the miniaturized hTEE probe to be even safer: we have seen no major complications in more than 200 hTEE examinations performed to date in our institution.

The small size and simplicity of the hTEE probe make it ideal for ECMO weaning because the entire weaning process may take several hours to observe hemodynamics after each adjustment of ECMO flow, volume status, and inotropic support. Motivated by TEE-guided weaning of cardiopulmonary bypass in the operating room, ${ }^{11}$ timely and accurate clinical decisions can be made by the intensivist for weaning ECMO and subsequent treatment without additional personnel. We reviewed our experience using hTEE in patients on ECMO to assess the functional status of the LV and RV in weaning ECMO before proceeding to the next level of care.

\section{METHODS}

\section{Patients}

Between July 2011 and June 2012, 21 consecutive patients (Table 1) underwent centrally or peripherally cannulated VA ECMO support longer than 48 hours for multiorgan failure with acute cardiogenic shock, defined as a rapid decline of hemodynamics requiring multiple inotropes to achieve a cardiac index of $2 \mathrm{~L} / \mathrm{min}$ per $\mathrm{m}^{2}$. Once recovered, all patients underwent hTEE-guided ECMO weaning using our institutional hemodynamic weaning protocol. Patient demographics, including etiology of shock, are described in Table 1. This study was approved by the local internal review board.

\section{ImaCor hTEE Probe}

The hTEE probe is a disposable flexible probe with a diameter of approximately $5.5 \mathrm{~mm}$, similar in size to an oral gastric tube, approved by the Food and Drug Administration for continuous cardiac function monitoring over a 72-hour period. The hTEE probe has $15 \mathrm{~cm}$ of penetration at $6.67 \mathrm{MHz}$ (B-mode) and can obtain images revealing left and right ventricular function and volume status. A midesophageal 4-chamber view was obtained, on average, 42 to $45 \mathrm{~cm}$ from the incisors. A short-axis transgastric view was obtained by manipulating the flexible tip of the probe using the hand control. Echo parameters obtainable by hTEE include dimension, area, and ratio measurements in the transgastric short-axis view and the midesophageal 4-chamber view, in particular, left ventricular systolic and diastolic area and left ventricular fractional area change. However, these quantitative values were not routinely recorded in our practice. Instead, images obtained at hTEE were used directly for clinical decision making because these qualitative assessments are well established and validated in hemodynamic management. ${ }^{4}$ All hTEE procedures were performed by cardiothoracic surgery intensivists, and none of them were TEE-trained cardiologists.

\section{ECMO Weaning Trial Protocol}

Before weaning ECMO, all patients met the criteria of being afebrile and euvolemic, with resolution of pulmonary edema on x-ray film, with adequate upper extremity arterial $\mathrm{PaO}_{2}$ and saturation on $50 \% \mathrm{FiO}_{2}$ from both the ECMO and ventilator circuits, and all pre-ECMO end-organ dysfunction recovered to baseline.

For the weaning trial, anticoagulation was titrated to a partial thromboplastin time target of 60 to 70 seconds to avoid thrombotic complications while decreasing ECMO flow over the period of assessment. Standard vital parameters (arterial blood pressure, central venous pressure, heart rate, and rhythm) were monitored; no Swan-Ganz catheter was inserted during the weaning process. The hTEE probe was inserted transorally in all patients. Additional sedation was not required because of the small size of the probe. The weaning trial consisted of 4 stages:

Stage 1: Baseline LV and RV functions and volume status were assessed by hTEE on full-flow ECMO support.

Stage 2: After assessment of baseline data, ECMO flow was gradually decreased in steps of $0.5-\mathrm{L} / \mathrm{min}$ increments to the goal of half of the original flow (stage 2). Throughout the weaning protocol, LV and RV function and hemodynamic responses (heart rate and blood pressure) were monitored continuously to allow assessment of ventricular function and volume status. If, at any period in the weaning protocol, LV or RV distension occurred or significant hypotension was observed, the weaning trial was aborted and ECMO support was returned to full flow.

Stage 3: Volume challenges were done with $5 \%$ albumin $(10 \mathrm{~mL} / \mathrm{kg})$ while ECMO flow was decreased to a minimum flow of 1.2 to $1.5 \mathrm{~L} / \mathrm{min}$. This volume challenge was necessary to achieve appropriate preload. Hemodynamic responses were monitored.

Stage 4: Inotropes (dobutamine and/or milrinone) were started, and the $\mathrm{LV}$ and RV functions and hemodynamics were observed for adequate response over 1 hour (a few hours for milrinone). If both LV and RV functions were recovered, the patient was considered for definitive ECMO removal. If LV dysfunction persisted, but RV function was recovered or improved, with inotropic support, the patient was considered for LVAD placement. If RV dysfunction persisted, but LV function was recovered, with inotropic support, the patient was considered for external right VAD placement. If biventricular dysfunction was observed, repeat assessment or total artificial heart placement was considered. If biventricular dysfunction was present and the patient was not a candidate for support because of another comorbid condition, end-of-life discussion was considered, because recovery is less likely.

After completion of the weaning trial, the ECMO flow was returned to full flow and the case was discussed with intensivists, surgeons, cardiologists, and family members for the timing of surgical intervention. A Swan-Ganz catheter was not used in any cases in the patients on ECMO because of a safety issue, such as migration of the catheter or introduction of the air to the ECMO system, and because of unreliability of the SwanGanz parameters because of the presence of suction applied on the venous cannula on the ECMO. The weaning processes are summarized in Table 2. 
TABLE 1. Patient demographics

\begin{tabular}{lc}
\hline \multicolumn{1}{c}{ Demographics } & Value (N= 21) \\
\hline Male, No. (\%) & $13(62)$ \\
Age, mean \pm SD (range), y & $49 \pm 12(26-61)$ \\
Duration of ECMO, mean \pm SD (range), d & $11.2 \pm 6.0(5-24)$ \\
Cannulation site, No. (\%) & \\
Peripheral cannulation & $19(91)$ \\
Central cannulation & $2(9)$ \\
Status at ECMO placement, No. (\%) & \\
Salvage procedure & $7(33)$ \\
Emergent procedure & $6(29)$ \\
Urgent procedure & $8(38)$ \\
Etiology of shock, No. (\%) & \\
Acutely decompensated cardiomyopathy & $10(48)$ \\
Acute myocarditis & $3(14)$ \\
Acute myocardial infarction & $3(14)$ \\
Postcardiotomy syndrome & $2(9.5)$ \\
Pulmonary embolism & $1(4.8)$ \\
Pulseless electronic activity code & $1(4.8)$ \\
Acute rejection & $1(4.8)$ \\
\hline$E C M O$, Extracorporeal membrane oxygenation, SD, standard deviation.
\end{tabular}

\section{RESULTS}

A total of $7(33 \%)$ of the patients did not tolerate the initial or subsequent weaning trials, and the procedure was aborted. These 7 patients remained in severe biventricular failure regardless of volume or inotropic challenge and were not candidates for either transplant or biventricular devices secondary to medical contraindications: ongoing sepsis $(n=2)$, documented peri-ECMO procedure stroke $(\mathrm{n}=3)$, and anoxic brain injury and family wanted to withdraw care $(\mathrm{n}=1)$. The weaning protocol was repeated in 2 to 3 days, and none of the patients demonstrated adequate cardiac recovery. These patients were given end-of-life care after discussion with family and palliative care consultants. The mean length of ECMO support for these 7 patients was $10.1 \pm 6.1$ (range, 6-24) days.

A total of $14(67 \%)$ of 21 patients tolerated the full weaning process (stage 1-4) with hTEE monitoring and evaluation. A total of $8(57 \%)$ of 14 patients had recoverable RV function with persistent LV dysfunction on the basis of the hTEE weaning protocol. All 8 patients were successfully bridged to LVAD within 1 to 4 days after the weaning trial. The typical pattern of hTEE findings in patients who underwent LVAD placement consisted of the presence of minor contractility of RV in stage 2, minimal distension of LV and RV on volume challenges (stage 3), appropriate RV contractility on stage 4 , and poor LV contractility with dilated LV in stage 4 . Among these 8 patients who underwent LVAD placement after ECMO weaning, 4 patients were discharged from the hospital, giving a survival rate of $50 \%$. Among the nonsurvivors, 3 died from postoperative non-device-related sepsis and
TABLE 2. VA ECMO weaning protocol using hTEE

\begin{tabular}{|c|c|}
\hline Stage & Actions \\
\hline Before weaning & $\begin{array}{l}\text { Prerequisite: patient is euvolemic and afebrile, chest } \\
\text { X-ray film is clear, and end-organ injury is } \\
\text { resolved. Increase heparinization for PTT goal of } \\
60-70 \text { seconds. } \\
\text { Insert hTEE probe. }\end{array}$ \\
\hline Stage 1 & $\begin{array}{l}\text { Baseline assessment of right and left ventricular } \\
\text { function with full ECMO flow. }\end{array}$ \\
\hline Stage 2 & $\begin{array}{l}\text { Decrease flow from full to half flow in increments of } \\
0.5 \mathrm{~L} / \mathrm{min} \text { and assess LV and RV function by hTEE } \\
\text { over at least } 1 / 2 \mathrm{~h} \text { after each decrease. If distention } \\
\text { occurs, return to full flow and abort trial. }\end{array}$ \\
\hline Stage 3 & $\begin{array}{l}\text { Volume load ( } 10 \mathrm{~mL} / \mathrm{kg}) \text { over } 20 \text { minutes, with half } \\
\text { flow, and assess RV and LV function by hTEE over } \\
\text { at least } 1 \mathrm{~h} \text {. }\end{array}$ \\
\hline Stage 4 & $\begin{array}{l}\text { Load inotrope (dobutamine and/or milrinone), } \\
\text { decrease flow to minimum (1-1.5/min), and assess } \\
\text { LV and RV function at least } 1 \mathrm{~h} \text {. }\end{array}$ \\
\hline $\begin{array}{c}\text { After weaning } \\
\text { assessment }\end{array}$ & $\begin{array}{l}\text { If biventricular failure persists, consider evaluation } \\
\text { for total artificial heart placement end-of-life } \\
\text { discussion. } \\
\text { If } \mathrm{LV} \text { dysfunction persists but RV function is } \\
\text { recovered, consider LVAD insertion. } \\
\text { If RV dysfunction persists but LV function is } \\
\text { recovered, consider external RVAD. } \\
\text { If both LV and RV functions are recovered, consider } \\
\text { ECMO decannulation. }\end{array}$ \\
\hline After weaning & $\begin{array}{l}\text { Return to full flow and discuss timing of surgical } \\
\text { intervention. }\end{array}$ \\
\hline
\end{tabular}

$\overline{V A \text {, Venoarterial; ECMO, extracorporeal membrane oxygenation; } h T E E \text {, hemodynamic }}$ transesophageal echocardiography; $P T T$, partial thromboplastin time; $L V$, left ventricle; $R V$, right ventricle; $L V A D$, left ventricular assist device; $R V A D$, right ventricular assist device.

1 from perioperative stroke. No patient died from RV failure or conditions related to ECMO. The 8 patients who received LVAD placement were on ECMO for $13.4 \pm 6.1$ (range, 5-23) days.

A total of $6(29 \%)$ of 14 patients had acceptable LV and $\mathrm{RV}$ recovery by hTEE-guided ECMO weaning. A total of 5 $(83 \%)$ of these 6 patients were decannulated without any other intervention. RV recovery was demonstrated in 1 patient who had ongoing reversible LV ischemia. This patient was able to be weaned off ECMO after conventional revascularization. One patient had biventricular recovery from ECMO; however, the patient had sustained an anoxic brain injury at ECMO cannulation secondary to prolonged code/hypoxia before ECMO placement. Although the patient was brain dead, circulation was maintained by ECMO; thus, the patient was able to donate the liver, kidney, and other organs. ECMO was discontinued at organ harvest. One patient was fully recovered from cardiogenic shock, weaned off ECMO support, and free of the inotropic support. However, the patient died from arrhythmia-related cardiac arrest after internal cardiac defibrillator placement 
while in-hospital. The mean length of ECMO support of these 6 patients who had LV and RV recovery was $10.3 \pm$ 4.3 (range, 5-17) days. Among these 6 patients who had recovered $\mathrm{LV}$ and $\mathrm{RV}$ functions, 4 were discharged from the hospital, giving a survival rate of $67 \%$.

Patterns of the ventricular recovery and failure are shown in Table 3. The final disposition of all the patients who underwent the weaning trial is illustrated in Figure 1. Overall, all 14 patients who passed the hTEE-guided ECMO weaning trial protocol were successfully weaned from ECMO, and none of the 7 patients who failed the weaning trial subsequently regained $\mathrm{RV}$ or left function.

The ability of hTEE to detect left or right ventricular recovery was statistically significant $(P<.0001$ by the Fisher exact test). The calculated sensitivity using 2-way contingency table analyses was $100 \%(95 \%$ confidence interval [CI], 73.2\%-100\%); specificity, $100 \%$ ( $95 \%$ CI, $56.1 \%-100 \%$ ); positive predictive value, $100 \%$ (95\% CI, $73.2 \%-100 \%)$; and negative predictive value, $100 \%(95 \%$ CI, $56.1 \%-100 \%)$. There were no hTEE probe-related complications observed during this study.

\section{DISCUSSION}

The management of patients on VA ECMO for cardiogenic shock is a clinical challenge for the intensivist regarding exit strategies. In far too many cases, the "crashand-burn" (INTERMACS 1) presentation of patients with viral cardiomyopathy, postmyocardial infarction, or cardiogenic shock requires urgent placement on VA ECMO. In that situation, clinicians may not have a clear knowledge of biventricular function and/or the patient. This uncertainty presents significant challenges for management after end-organ and neurologic function recovery. ECMO can certainly buy time to make a decision, and a recent review of our ECMO series found ECMO improved end-organ functions. ${ }^{12}$

When RV failure exists in the setting of an LVAD implantation, the perioperative mortality rate increases up to $19 \%$ to $43 \% .{ }^{13}$ Failure to recognize a dysfunctional $\mathrm{RV}$ on transition to an LVAD may result in a protracted intensive care unit (ICU) course to support a borderline right ventricle or emergent placement of an RVAD. ${ }^{14}$
The duration of ECMO support and transition to a VAD bridge have been poorly defined. Most ECMO centers perform ECMO weaning without clear guidelines or algorithms. ${ }^{15}$ Premature withdrawal from ECMO support without sufficient $\mathrm{RV}$ recovery runs the risk of the scenario previously described. Unnecessary delays in the weaning process increase the chance of complications from prolonged ECMO support and the ICU cost. In addition, continuing prolonged ECMO support on a patient with no evidence of any ventricular recovery on repeated weaning studies and no option for LVAD or biventricular assist device bridging would not be cost effective and would be ethically questionable. For patients demonstrating biventricular failure after repeated attempts at weaning, families may be able to use this information as guidance for end-of-life planning.

Conventional determination of cardiac output using the Swan-Ganz catheter via traditional thermodilution is of limited value in patients on ECMO ${ }^{16}$ Minimally invasive measurement of cardiac output via pulse contour, esophageal Doppler, partial carbon dioxide rebreathing, and thoracic bioimpedance of the thoracic aorta is similarly not reliable in the setting of VA ECMO. ${ }^{17,18}$ Serial mixed venous oxygen saturation assessments require a Swan-Ganz catheter, and there are always time gaps between samplings, which do not reflect real-time dynamic changes of the ventricle during ECMO weaning. Arterial waveform analyses during the ECMO weaning may be helpful to determine the ejection of the heart; however, it does not directly reflect cardiac volume status. No other technology exists that can be used at the bedside by the cardiac intensivist for any duration of time and at any time of day for accurate monitoring of the ECMO patient. ${ }^{19}$ TEE has been used as a gold standard for cardiac monitoring in the operating room in patients undergoing cardiac surgery, in particular for weaning of cardiopulmonary bypass. ${ }^{11,20}$

In the ICU, the TEE could be an ideal modality for ECMO weaning because TEE would provide accurate information for ventricular filling and function. Rastan and colleagues ${ }^{21}$ reported more than 500 ECMO cases for postcardiotomy cardiogenic shock with a relatively short

TABLE 3. Pattern of ventricular recovery and failure

\begin{tabular}{|c|c|c|c|c|}
\hline \multirow[b]{2}{*}{ Stage } & \multicolumn{2}{|c|}{ Ventricular recovery } & \multicolumn{2}{|c|}{ Ventricular failure } \\
\hline & Contractility & Volume & Contractility & Volume \\
\hline Stage 1 (baseline) & Baseline & Decompressed & Baseline & Decompressed \\
\hline Stage 2 (half flow) & $\begin{array}{l}\text { Beginning to respond to } \\
\text { reduced ECMO flow }\end{array}$ & $\begin{array}{l}\text { Adequate volume in } \\
\text { ventricle }\end{array}$ & Same as stage 1 (no response) & Mild distension \\
\hline $\begin{array}{l}\text { Stage } 3 \text { (volume challenge } \\
\text { and low ECMO flow) }\end{array}$ & $\begin{array}{l}\text { Same as stage } 2 \text { or mild } \\
\text { contractility }\end{array}$ & $\begin{array}{l}\text { Adequate response to } \\
\text { volume }\end{array}$ & Same as stage 1 (no response) & $\begin{array}{l}\text { Increase distension } \\
\quad \text { (consider termination) }\end{array}$ \\
\hline Stage 4 (inotropes) & Adequate contractility & Euvolemic & No response to inotropes & $\begin{array}{l}\text { Distended ventricle } \\
\text { (termination) }\end{array}$ \\
\hline
\end{tabular}

ECMO, Extracorporeal membrane oxygenation. 


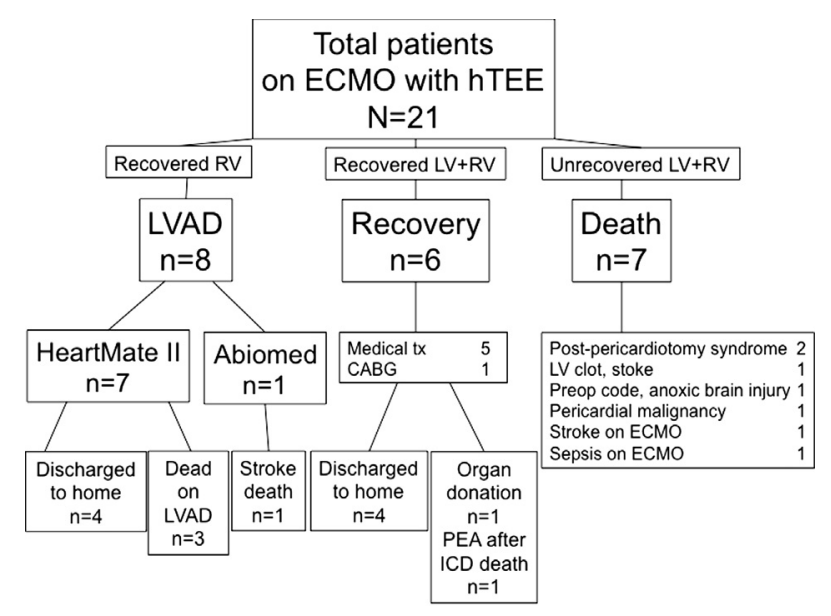

FIGURE 1. The final disposition of patients on the basis of hemodynamic transesophageal echocardiography ( $h T E E)$ assessment. ECMO, Extracorporeal membrane oxygenation; $R V$, right ventricle; $L V$, left ventricle; $L V A D$, left ventricular assist device; $t x$, treatment; $C A B G$, coronary artery bypass grafting; PEA, pulseless electric activity; $I C D$, internal cardiac defibrillator.

ECMO support period (mean ECMO support, 3.3 days). Their ECMO weaning was initiated a few days after ECMO placement, and they evaluated the LV and RV function by conventional TEE performed a few times a day. ${ }^{21}$ Two other groups reported qualitative TEE assessment in ECMO weaning without specific protocol. $^{22,23}$

In a practical hospital setting, TEE services have significant limitations regarding their availability because of the requirement of certified personnel and equipment. ${ }^{19,22}$ The expense and time commitment from the TEE service dedicated to full weaning assessment at the bedside would be difficult. Transthoracic echocardiography (TTE) can be performed without limitation of personnel; however, the visualization of the ventricle could be limited by patient body habitus and/or technician's ability. ${ }^{7}$ Furthermore, both TEE and TTE share the same problem: conventional TEE and TTE studies represent only a single "snapshot" in time.

Our weaning protocol itself is a bedside analog of weaning from cardiopulmonary bypass in the operating room after cardiac surgery, with one key addition: prolonged time of observation. The hTEE monitoring was able to accurately reveal the volume response to fluid challenge and the functional response to inotropes. In the early period of study, we performed parallel echocardiography, and these results were the same as the hTEE; thus, parallel studies are no longer performed for the ECMO weaning. The hTEE in our institution was performed by the qualified cardiac surgery intensivists (N.C.C., H.T.P., Q.Y., and H.H.). All images were reviewed by cardiac surgeons and cardiologists before making final decisions.
The hTEE training consisted of didactic education mannequin training and bedside demonstration of at least 10 cases with experienced individuals.

We, therefore, developed an hTEE-based weaning protocol to make a real-time assessment of both LV and RV function throughout the ECMO weaning process. Furthermore, we chose a qualitative approach, which involves visual assessment of RV and LV function and filling, instead of previous quantitative approaches. ${ }^{22,23}$ The validity of qualitative assessment has been well established and is readily learned. ${ }^{4,24}$

The weaning process requires a staged approach with decreased ECMO support, volume loading, and inotrope support over 4 to 6 hours. Although such a weaning trial can be performed in the operating room using conventional TEE, this is not practical because of time constraints. The miniaturized hTEE probe offers ease of insertion and reproducibility of the assessment of both LV and RV volume status and contractility, and allows continuous monitoring throughout the weaning trial. The quality and reliability of the information obtained from hTEE regarding ventricular function and volume status appears to be comparable to conventional TEE. ${ }^{5,6}$ There was excellent correlation with TTE done at the end of the weaning process to confirm hTEE findings.

The small size of the hTEE probe may further reduce complications associated with multiple conventional TEEs, which are reported to occur $0.2 \%$ to $0.5 \%$ per insertion, with an estimated mortality of less than $0.01 \%$ per insertion. ${ }^{7-10}$ In our limited experience of hTEE over 1 year, we had performed more than 200 studies without hTEE probe-related complications. Our hTEE-guided weaning protocol has accurately predicted successful weaning off ECMO, or bridging from ECMO to LVAD. In our study, no patients predicted to have a recovered RV function had any significant right ventricular failure after LVAD insertion. No patients weaned to medical management off ECMO on the basis of the weaning trial protocol required any further mechanical, significant, or prolonged pharmacologic support. The sensitivity and specificity of the hTEE for RV recovery were excellent; however, a larger study is needed to reduce the size of CIs for positive and negative predictive values.

This study was limited, in part, by the relatively few patients. Our ECMO program was newly established in 2011; thus, we do not have any data to compare the results of ECMO weaning using hTEE with other conventional monitoring methods.

\section{CONCLUSIONS}

In our experience with 21 patients weaned from VA ECMO using the hTEE-based protocol, hTEE accurately predicted therapeutic outcomes. During this weaning process, no patient developed complications related to the 
weaning process or hTEE itself. Of those that the hTEE demonstrated RV recovery, but ongoing LV dysfunction, all were successfully weaned from ECMO and underwent LVAD implantation without any significant RV dysfunction. Of the patients demonstrating biventricular recovery weaned, they had a reasonable clinical outcome after ECMO decannulation. In cases of repeated biventricular failure in attempts at weaning, with significant comorbidities prohibiting further transplantation or an implantable biventricular device, families were able to use the information to help guide their decision to withdraw. The hTEE-based weaning protocol was simple and easily reproducible by the intensivist assessing the patient.

\section{References}

1. Hastings HM. Transesophageal echocardiography-guided hemodynamic assessment and management. ICU Director. 2012;3:38-41.

2. Geisen M, Caliandro F, Edsell ME. Initial clinical experience with a novel, miniaturized transoesophageal echocardiography probe. Intensive Care Med. 2012;38(suppl 1). Abstract 0272

3. Merz TM, Cioccari L, Baur H-R, Takala J. Management of hemodynamically compromised patients using the ImaCor Claritee probe: a feasibility study. Intensive Care Med. 2012;38(suppl 1). Abstract 0991.

4. Benjamin E, Griffin K, Leibowitz AB, Manasia A, Oropello JM, Geffroy V, et al. Goal-directed transesophageal echocardiography performed by intensivists to assess left ventricular function: comparison with pulmonary artery catheterization. J Cardiothorac Vasc Anesth. 1998;12:10-5.

5. Vieillard-Baron A, Slama M, Mayo P, Charron C, Amiel JB, Esterez C, et al. A pilot study on safety and clinical utility of a single-use 72-hour indwelling transesophageal echocardiography probe. Intensive Care Med. 2013;39:629-35.

6. Hüttemann E. Transoesophageal echocardiography in critical care. Minerva Anestesiol. 2006;72:891-913.

7. Hilberath JN, Oakes DA, Shernan SK, Bulwer BE, D'Ambra MN, Eltzschig HK. Safety of transesophageal echocardiography. $J$ Am Soc Echocardiogr. 2010;23: 1115-27.

8. Daniel WG, Erbel R, Kasper W, Visser CA, Engberding R, Sutherland GR, et al. Safety of transesophageal echocardiography: a multicenter survey of 10,419 examinations. Circulation. 1991;83:817-21.

9. Kallmeyer IJ, Collard CD, Fox JA, Body SC, Shernan SK. The safety of intraoperative transesophageal echocardiography: a case series of 7200 cardiac surgical patients. Anesth Analg. 2001;92:1126-30.
10. Min JK, Spencer KT, Furlong KT, DeCara JM, Sugeng L, Ward RP, et al Clinical features of complications from transesophageal echocardiography: a single-center case series of 10,000 consecutive examinations. J Am Soc Echocardiogr. 2005; 18:925-9.

11. Licker M, Diaper J, Cartier V, Ellenberger C, Cikirikcioglu M, Kalangos A, et al. Clinical review: management of weaning from cardiopulmonary bypass after cardiac surgery. Ann Card Anaesth. 2012;15:206-23.

12. Wong JK, Siow VS, Hirose H, Karbowski P, Miessau J, Baram M, et al. End organ recovery and survival with the QuadroxD oxygenator in adults on extracorporeal membrane oxygenation. World J Cardiovasc Surg. 2012;2:73-80.

13. Ochiai Y, McCarthy PM, Smedira NG, Banbury MK, Navia JL, Feng J, et al Predictors of severe right ventricular failure after implantable left ventricular assist device insertion: analysis of 245 patients. Circulation. 2002; 106:I198-202

14. Pettinari M, Jacobs S, Rega F, Verbelen T, Droogne W, Meyns B. Are right ventricular risk scores useful? Eur J Cardiothorac Surg. 2012;42:621-6.

15. Fiser SM, Tribble CG, Kaza AK, Long SM, Zacour RK, Kern JA, et al. When to discontinue extracorporeal membrane oxygenation for postcardiotomy support. Ann Thorac Surg. 2001;71:210-4.

16. Lee AJ, Cohn JH, Ranasinghe JS. Cardiac output assessed by invasive and minimally invasive techniques. Anesthesiol Res Pract. 2011;2011:475151.

17. Peyton PJ, Chong SW. Minimally invasive measurement of cardiac output during surgery and critical care: a meta-analysis of accuracy and precision. Anesthesiology. 2010;113:1220-35.

18. Spiess BD, Patel MA, Soltow LO, Wright IH. Comparison of bioimpedance versus thermodilution cardiac output during cardiac surgery: evaluation of a second-generation bioimpedance device. J Cardiothorac Vasc Anesth. 2001;15 567-73.

19. Greenhalgh DL, Patrick MR. Perioperative transesophageal echocardiography: past, present \& future. Anesthesia. 2012;67:343-6.

20. Vincent JL, Rhodes A, Perel A, Martin GS, Della Rocca G, Vallet B, et al Clinical review: update on hemodynamic monitoring-a consensus of 16 Crit Care. 2011;15:229.

21. Rastan AJ, Dege A, Mohr M, Doll N, Falk V, Walther T, et al. Early and late outcomes of 517 consecutive adult patients treated with extracorporeal membrane oxygenation for refractory postcardiotomy cardiogenic shock. J Thorac Cardiovasc Surg. 2010;139:302-11.

22. Aissaoui N, Luyt CE, Leprince P, Trouillet JL, Léger P, Pavie A, et al. Predictors of successful extracorporeal membrane oxygenation (ECMO) weaning after assistance for refractory cardiogenic shock. Intensive Care Med. 2011;37: $1738-45$.

23. Platts DG, Sedgwick JF, Burstow DJ, Mullany DV, Fraser JF. The role of echocardiography in the management of patients supported by extracorporeal membrane oxygenation. J Am Soc Echocardiogr. 2012;25:131-41.

24. Vieillard-Baron A, Charron C, Chergui K, Peyrouset O, Jardin F. Bedside echocardiographic evaluation of hemodynamics in sepsis: is a qualitative evaluation sufficient? Intensive Care Med. 2006;32:1547-52. 